\title{
Chemical composition and thermal properties of Pistacia atlantica subsp. Kurdica gum
}

Fardin Mirahmadi ${ }^{1}$, Maryam Mizani ${ }^{*}$, Rahmat Sadeghi ${ }^{2}$ and Mohammad Hadi Givianrad ${ }^{3}$

\begin{abstract}
Pistacia atlantica subsp. Kurdica (PAK) is distributed throughout the Zagros Mountains and is indigenous to Kurdistan province in western Iran. This study focused on the composition and thermal properties of gum extracted from female and male trees from six regions of Kurdistan province. Significant differences were detected in the total protein, total ash, total carbohydrate and monosaccharaide contents according to gender and geographic region, but no significant difference was found for moisture content. Analysis of the monosaccharide composition using HPLC showed the presence of arabinose, galactose, glucose, rhamnose and xylose. Significant differences were observed for the amino acid contents of the various PAK gum samples. The most abundant amino acids were glutamic acid, aspartic acid, serine, proline and histidine; however, the relative proportions of amino acids varied considerably between samples. The results indicate that the volatile components $(\mathrm{VoC})$ were significantly different between samples according to gender and region, with the predominant $\mathrm{VoC}$ being a-Pinene. The results of thermogravimetric analysis showed that the onset of the initial and main decomposition of the samples was at $80^{\circ} \mathrm{C}$ and above $240{ }^{\circ} \mathrm{C}$, respectively. The differential scanning calorimetry results showed that nearly all gum samples included two glass transition temperatures and heat capacity values and that nearly all of the values for the female gum samples were lower than for the male samples.
\end{abstract}

Keywords: Chemical composition, Gum, Pistacia atlantica subsp. Kurdica, Thermal properties

\section{Introduction}

There are 11 species of Pistacia globally [1]. Pistacia atlantica is widely distributed from the Canary Mountains in Spain to the mountains of Iran [2]. Iran is a major source of Pistacia diversity in pistachio cultivation and production [3]. P. atlantica has four subspecies (mutica, cubolica, atlantica and kurdica) [4]. P. atlantica subsp. Kurdica (PAK) is distributed throughout the Zagros Mountains and is indigenous to Kurdistan province in western Iran [5].

Herbal medicine has been used to treat diseases because of its therapeutic potential [6]. The different products of PAK (leaves, fruit and gum) are valuable for

\footnotetext{
*Correspondence: mmizani2000@gmail.com; m.mizani@srbiau.ac.ir ${ }^{1}$ Department of Food Science and Technology, Science and Research Branch, Islamic Azad University, Tehran, Iran

Full list of author information is available at the end of the article
}

their medicinal, cosmetic and food value. They are used to treat digestive disturbances and as moisturizers, antimicrobials, antioxidants, edible oil, feed and chewing gum $[7,8]$. The quality and quantity of the gum, or oleoresin (jajka talla in Kurdish), depends on the species of plant, gender and geographic origin [9]. Several studies have examined the chemical composition of $P$. atlantica essential oil $[4,10]$. The optical rotation and nitrogen, ash, moisture and tannin contents are the parameters that are frequently used to recognize the properties of gums used as food additives. The unique fundamental properties of this gum are its high water solubility and low viscosity [11].

Knowledge about the thermal properties of food and food components is important. Thermogravimetric analysis (TGA) continuously determines the weight of a sample as a function of temperature over time. It detects changes in the mass of a sample (gain or loss) 
and determines the temperatures that characterize a step in mass loss and gain curves to monitor food processes [12]. Several researchers have used thermal properties to calculate the energy of activation based on the Broido model. An increase in the energy of activation indicates a higher thermal stability, so that an activation energy of above 100 represents high polymer stability and suggests the presence of synthetic polymers as compared to the lower activation energy of biopolymers. The difference in the energy of activation can be influenced by the origin and species of the gum [10].

Differential scanning calorimetry (DSC) measures the heat flow of samples as a function of temperature or time. This method determines physical transition and chemical reactions quantitatively [13]. The glass transition temperatures $\left(T_{g}\right)$ of synthetic polymers and biopolymers have been measured using DSC. At the glass transition temperature, materials convert from very viscous glassy to rubbery form, the molecular mobility increases and the viscosity decreases. This may result in structural changes such as stickiness and collapse [14].

\section{Materials and methods Gum extraction}

The oleoresin of PAK was extracted by piercing the trunk of the tree with a specialized tool and collected in a clay bowl attached to the trunk [15]. To keep minimum damage during oeloresin extraction and maximum consistency among samples, a sampling strategy introduced by Karamshahi et al. [16] was employed. Trees with $30-50 \mathrm{~cm}$ trunk diameter were selected and 20 horizontal grooves $(2 \mathrm{~cm}$ length) were pierced to collect the resinous exudates from male and female trees at the beginning of July 2016 in six regions of Kurdistan province in Iran (Armardeh, Kanisoor, Marivan, Sarvabad, Dezli, Hawraman).

\section{Moisture, total ash and protein contents}

The moisture and total ash contents of the oleoresin samples were determined using the AOAC method [17]. The total protein content was determined by analyzing the nitrogen content using the semi-micro Khjeldahl method [17]. On the basis of early determinations, a conversion factor of 6.6 was used to calculate the crude protein content [18].

\section{Total carbohydrate content}

In order to determine the total carbohydrates (TC) content, $4 \mathrm{~g}$ of crude gum was dissolved in a final volume of $100 \mathrm{~mL}$ of distilled water at $50{ }^{\circ} \mathrm{C}$ and then filtered through Whatman no. 1 filter paper [19]. The total carbohydrate content of the $4 \%$ solution was determined by the phenol-sulfuric acid colorimetric method with D-glucose as the standard at $490 \mathrm{~nm}$ [20].

\section{Amino acid composition}

Oleoresin samples were hydrolyzed with $0.2 \mathrm{~mL}$ of $5.5 \mathrm{~N}$ in constantly boiling $\mathrm{HCl}$ in a sealed tube at $110{ }^{\circ} \mathrm{C}$ for $18 \mathrm{~h} \mathrm{[21].} \mathrm{The} \mathrm{hydrolyzed} \mathrm{liquid} \mathrm{was} \mathrm{neutralized} \mathrm{with}$ bicarbonate and centrifuged at 13,500 $g$ for $10 \mathrm{~min}$. Then $200 \mu \mathrm{L}$ of the supernatant was mixed with $50 \mu \mathrm{L}$ of 4-(4,dimethylamino) phenyl azobenzene sulfonyl chloride $\left(20 \mathrm{mg} \mathrm{mL}^{-1}\right)$ at $60{ }^{\circ} \mathrm{C}$ for $60 \mathrm{~min}$ to convert the amino acids into dabsyl chloride derivatives. The acetone then was evaporated and the final solution was injected into the HPLC $[21,22]$.

\section{Monosaccharide composition}

The sample was labeled by adding $30 \mu \mathrm{L}$ of $\mathrm{NaOH}(0.3 \mathrm{M})$ and $20 \mu \mathrm{L}$ of PMP (1-phenyl-3-methyl-5-pyrazolone) solution (0.5 M in methanol). Fucose was added as an internal standard to each sample before derivatization. The mixtures were incubated at $70{ }^{\circ} \mathrm{C}$ for $60 \mathrm{~min}$, cooled to room temperature and neutralized with $30 \mu \mathrm{L}$ of $\mathrm{HCl}$ $(0.3 \mathrm{M}), 1 \mathrm{~mL}$ of trichloromethane was added. After vigorous shaking and layering, the organic phase was carefully removed and discarded. The aqueous layer was passed through a $0.45 \mu \mathrm{m}$ syringe filter before HPLC analysis.

The PMP-labeled monosaccharides were analyzed using an Unicam Crystal 200 HPLC system consisting of a G1311C quaternary pump, G1329B autosampler $(0.1-100 \mu \mathrm{L}), \mathrm{G} 1316 \mathrm{~A}$ pre-column oven $(273-333 \mathrm{~K})$ and G1315D-DAD detector (190-950 nm). The analytical column was a TC-C18 column $(4.6 \times 250 \mathrm{~mm}$; $5 \mu \mathrm{m})$. The injection volume was $20 \mu \mathrm{L}$ with an eluent flow rate of $1.0 \mathrm{~mL} \mathrm{~min}{ }^{-1}$ at $35^{\circ} \mathrm{C}$. Mobile phase A was $100 \%$ acetonitrile and mobile phase B was a mixture of distilled water and acetonitrile (90: 10, v/v) with $0.045 \%$ $\mathrm{KH}_{2} \mathrm{PO}_{4}-0.05 \%$ triethylamine buffer $(\mathrm{pH} 7.5)$. Gradient elution was performed at $94 \%-94 \%-88 \%-88 \%$ B with linear decreases at 0-4-5-20 min. The UV detection wavelength was $245 \mathrm{~nm}$ [23].

\section{Volatile components}

To prepare the sample extracts, the oleoresin (75 g) was firstly extracted with $300 \mathrm{~mL}$ of ethanol (90\%) by sonication. The $\mathrm{n}$-hexane fraction was saponified with $0.5 \mathrm{~N}$ methanolic $\mathrm{NaOH}$ solution by heating in a steam bath until fat globules entered the solution (approximately $5 \mathrm{~min}$ ) and then boiled for $2 \mathrm{~min}$. After cooling, saturated $\mathrm{NaCl}$ solution was added to each solution. The mixtures were transferred to a separatory funnel individually and each was extracted with $30 \mathrm{~mL}$ of petroleum ether and 
converted to their methyl ester forms with $20 \mathrm{~mL}$ of boron trifluoride-methanol complex reagent [6].

Chromatographic analysis was carried out on Agilent 6890N network GC system combined with an Agilent 5973 network mass selective detector (GC-MS). The capillary column used was an Agilent 19091N-136 (HP Innowax capillary: $60.0 \mathrm{~m} \times 0.25 \mathrm{~mm} \times 0.25 \mu \mathrm{m})$. Helium was used as a carrier gas at a flow rate of $0.8 \mathrm{~mL} \mathrm{~min} \mathrm{~m}^{-1}$ with a 11 injection volume. Samples were analyzed with the column held initially at $60^{\circ} \mathrm{C}$ for $1 \mathrm{~min}$ after injection with a $10 \mathrm{~min}$ hold time, then increased to $220^{\circ} \mathrm{C}$ with $4{ }^{\circ} \mathrm{C}$ min $^{-1}$ heating ramp and kept at $220{ }^{\circ} \mathrm{C}$ for $10 \mathrm{~min}$. The final temperature was increased to $240{ }^{\circ} \mathrm{C}$ with $1{ }^{\circ} \mathrm{C} \mathrm{min}{ }^{-1}$ heating ramp. The injection was performed in splitless mode. Detector and injector temperatures were 280 and $250{ }^{\circ} \mathrm{C}$, respectively. The run time was $80 \mathrm{~min}$ [6].

\section{Fourier transform infrared spectroscopy}

The IR spectra of the oleoresin samples were determined using Fourier transform infrared spectrometry (FTIR) (Perkin-Elmer; 16 PC spectrometer; USA). The samples were ground with spectroscopic grade potassium bromide powder and then pressed into $1 \mathrm{~mm}$ pellets for FTIR measurement at wavenumber ranges of 400 and $4000 \mathrm{~cm}^{-1}$ [24].

\section{Thermogravimetric analysis}

The thermal behavior of the oleoresin samples was analyzed by TGA (Mettler Toledo; TGA1, RT-800, STAR SW 10.00) at a heating rate of $10{ }^{\circ} \mathrm{C} \mathrm{min}-1$ at a temperature range of $0-600{ }^{\circ} \mathrm{C}$ in air. Samples of about $10 \mathrm{mg}$ were weighed in an aluminum pan. Derivatograms of the TGA curves (derivative mass loss) were also recorded [25].

\section{Differential scanning calorimetry}

DSC measurement was carried out in $\mathrm{N}_{2}$ atmosphere (Mettler Toledo; DSC1, - 130 to 600, STAR SW 12.00; Switzerland) calibrated with indium as a standard. Analysis started at $-120{ }^{\circ} \mathrm{C}$ and continued up to $80{ }^{\circ} \mathrm{C}$ at a heating rate of $10{ }^{\circ} \mathrm{C} \mathrm{min}{ }^{-1}$. Samples were weighed (10-15 mg) in aluminum DSC pans (aluminum crucible $=40 \mu \mathrm{L}$ ) and an empty pan was used as a Ref. [13].

\section{Statistical analysis}

All chemical analyses were performed in triplicate. All data was analyzed using a two-way general liner model design analysis of variance (ANOVA) with the factors of geographic region and gender. Tukey's multiple range test was used to compare the treatment means (SAS 9.3). Non-parametric Spearman rank correlation coefficients of chemical components and environment characteristics were calculated using the SPSS software version 22.0.

\section{Results and discussion \\ Chemical composition analysis}

The results of compositional analysis for all PAK oleoresin samples are summarized in the Tables 1, 2 and 3 . Significant differences were observed in the total protein $(P<0.01)$ and total ash $(P<0.05)$ contents for gender and geographic region, but no significant difference in moisture content was found. The values for moisture was $16.03 \%$ to $19.26 \%$, for total ash was $0.86 \%$ to $0.94 \%$ and for protein was $3.97 \%$ to $5.05 \%$.

Table 1 Chemical composition of the extracted gum samples for factors under investigation

\begin{tabular}{|c|c|c|c|c|c|c|c|c|}
\hline \multirow[t]{2}{*}{ Sample } & \multicolumn{2}{|l|}{ Gender } & \multicolumn{6}{|c|}{ Geographic regions } \\
\hline & Female & Male & Kanisoor & Armardeh & Hawraman & Dezli & Sarvabad & Marivan \\
\hline Moisture (\%) & $18.30 \pm 1.28 \mathrm{a}$ & $18.60 \pm 1.31 \mathrm{a}$ & $18.81 \pm 0.93 \mathrm{a}$ & $18.89 \pm 1.35 \mathrm{a}$ & $18.58 \pm 0.50 \mathrm{a}$ & $16.03 \pm 0.19 a$ & $19.12 \pm 2.20 \mathrm{a}$ & $19.26 \pm 0.16 a$ \\
\hline Ash (\%) & $0.93 \pm 0.002 \mathrm{a}$ & $0.88 \pm 0.003 b$ & $0.94 \pm 0.002 \mathrm{a}$ & $0.92 \pm 0.003 \mathrm{ab}$ & $0.86 \pm 0.003 b$ & $0.91 \pm 0.003 \mathrm{ab}$ & $0.93 \pm 0.002 \mathrm{ab}$ & $0.87 \pm 0.002 \mathrm{ab}$ \\
\hline $\begin{array}{l}\text { Totalcarbohy- } \\
\text { drates (\%) }\end{array}$ & $40.51 \pm 2.35 b$ & $44.74 \pm 2.53 \mathrm{a}$ & $45.56 \pm 2.12 \mathrm{a}$ & $41.23 \pm 3.04 \mathrm{a}$ & $43.36 \pm 1.63 \mathrm{a}$ & $44.01 \pm 2.63 \mathrm{a}$ & $39.83 \pm 1.33$ a & $41.76 \pm 1.76 \mathrm{a}$ \\
\hline Arabinose $^{a}$ & $73.63 \pm 6.09 a$ & $66.88 \pm 5.32 b$ & $78.00 \pm 4.13 b$ & $74.00 \pm 0.85 c$ & $60.50 \pm 1.51 \mathrm{e}$ & $69.00 \pm 0.85 d$ & $53.50 \pm 1.66 f$ & $86.50 \pm 2.20 \mathrm{a}$ \\
\hline Galactose & $53.42 \pm 5.55 \mathrm{a}$ & $49.42 \pm 5,66 \mathrm{~b}$ & $50.00 \pm 2.47 d$ & $69.00 \pm 0.97 a$ & $36.50 \pm 0.69 f$ & $59.63 \pm 0.81 b$ & $41.38 \pm 1.38 \mathrm{e}$ & $52.00 \pm 1.38 c$ \\
\hline Rhamnose & $20.96 \pm 3.18 \mathrm{a}$ & $19.08 \pm 2.47 b$ & $20.33 \pm 0.40 c$ & $13.08 \pm 0.48 f$ & $28.85 \pm 1.50 \mathrm{a}$ & $17.63 \pm 0.29 d$ & $24.15 \pm 0.93 b$ & $15.40 \pm 0.31 \mathrm{e}$ \\
\hline Fucose & $3.58 \pm 1.45 \mathrm{a}$ & $3.27 \pm 1.06 \mathrm{~b}$ & $1.73 \pm 0.33 d$ & $1.38 \pm 0.01 \mathrm{e}$ & $6.15 \pm 0.49 b$ & $2.37 \pm 0.15 c$ & $7.43 \pm 0.32 \mathrm{a}$ & $1.49 \pm 0.10 \mathrm{e}$ \\
\hline Xylose & $24.00 \pm 4.98 b$ & $25.5 \pm 4.35 a$ & $21.50 \pm 1.16 c$ & $17.00 \pm 0.38 \mathrm{e}$ & $36.50 \pm 0.59 b$ & $19.00 \pm 0.38 d$ & $38.00 \pm 0.85 a$ & $16.50 \pm 0.59 \mathrm{e}$ \\
\hline Glucose & $5.07 \pm 1.05 \mathrm{a}$ & $4.69 \pm 1.25 b$ & $4.63 \pm 0.13 c$ & $2.40 \pm 0.19 e$ & $7.58 \pm 0.29 b$ & $3.35 \pm 0.14 d$ & $8.08 \pm 0.35 a$ & $3.25 \pm 0.49 d$ \\
\hline Glucuronic acid & $22.88 \pm 2.78 \mathrm{a}$ & $17.47 \pm 2.77$ b & $15.30 \pm 0.59 \mathrm{e}$ & $15.70 \pm 2.99 \mathrm{e}$ & $23.33 \pm 2.03 b$ & $30.13 \pm 1.45 \mathrm{a}$ & $19.60 \pm 0.85 c$ & $16.50 \pm 0.23 f$ \\
\hline $\begin{array}{l}\text { Galacturonic } \\
\text { acid }\end{array}$ & $43.63 \pm 5.37 a$ & $35.33 \pm 4.98 b$ & $34.23 \pm 1.69 \mathrm{~d}$ & $49.80 \pm 2.58 b$ & $27.80 \pm 0.52 \mathrm{e}$ & $28.30 \pm 3.18 \mathrm{e}$ & $44.75 \pm 4.11 c$ & $52.00 \pm 1.39 a$ \\
\hline
\end{tabular}

Values with different letters are significantly different

a Unit for monosaccharide: milligrams (mg) per gram of carbohydrate 
Table 2 Chemical composition (total Protein and amino acids) of the extracted gum samples for factors under investigation

\begin{tabular}{|c|c|c|c|c|c|c|c|c|}
\hline \multirow[t]{2}{*}{ Sample } & \multicolumn{2}{|l|}{ Gender } & \multicolumn{6}{|c|}{ Geographic regions } \\
\hline & Female & Male & Kanisoor & Armardeh & Hawraman & Dezli & Sarvabad & Marivan \\
\hline $\begin{array}{l}\text { Total protein } \\
(\%)\end{array}$ & $63 \pm 0.22 a$ & $4.23 \pm 0.20 b$ & $4.09 \pm 0.12 \mathrm{~d}$ & $4.82 \pm 0.15 b$ & $4.18 \pm 0.05 d$ & $4.47 \pm 0.15 c$ & $5.05 \pm 0.13 a$ & $3.97 \pm 0.11 \mathrm{e}$ \\
\hline Alanine $^{a}$ & $46.17 \pm 5.37 \mathrm{a}$ & $35.33 \pm 4.98 b$ & $50.00 \pm 0.35 b$ & $40.63 \pm 7.60 c$ & $33.88 \pm 1.86 \mathrm{e}$ & $53.00 \pm 5.76 a$ & $37.63 \pm 2.82 d$ & $29.38 \pm 3.23 f$ \\
\hline Glycine & $40.29 \pm 5.68 b$ & $42.38 \pm 5.83 \mathrm{a}$ & $37.00 \pm 0.43 d$ & $32.50 \pm 0.59 \mathrm{e}$ & $59.00 \pm 0.89 a$ & $25.50 \pm 0.53 f$ & $43.5 \pm 0.59 c$ & $50.50 \pm 0.55 b$ \\
\hline Lysine & $41.75 \pm 1.87 b$ & $46.33 \pm 2.88 \mathrm{a}$ & $43.85 \pm 0.43 c d$ & $47.60 \pm 5.75 \mathrm{a}$ & $44.18 \pm 1.61 b c$ & $43.57 \pm 1.93 \mathrm{~d}$ & $44.30 \pm 0.68 b$ & $40.75 \pm 1.45 \mathrm{e}$ \\
\hline Cysteine & $4.16 \pm 0.88 \mathrm{a}$ & $3.42 \pm 1.43 b$ & $2.98 \pm 0.52 \mathrm{~d}$ & $4.05 \pm 0.02 c$ & $1.60 \pm 0.74 \mathrm{e}$ & $5.93 \pm 0.56 b$ & $7.03 \pm 0.65 \mathrm{a}$ & $1.15 \pm 0.53 f$ \\
\hline Threonine & $35.29 \pm 4.88 b$ & $47.39 \pm 6.23 \mathrm{a}$ & $50.25 \pm 0.21 b$ & $40.80 \pm 8.06 c$ & $34.13 \pm 1.72 \mathrm{e}$ & $55.38 \pm 6.51 \mathrm{a}$ & $38.05 \pm 3.26 \mathrm{~d}$ & $29.45 \pm 3.65 f$ \\
\hline Valine & $34.02 \pm 5.14 \mathrm{a}$ & $31.98 \pm 4.98 b$ & $48.75 \pm 0.75 a$ & $42.00 \pm 0.85 b$ & $33.25 \pm 0.44 c$ & $25.13 \pm 0.62 \mathrm{e}$ & $19.90 \pm 0.55 f$ & $29.00 \pm 0.35 d$ \\
\hline Phenylalanine & $24.71 \pm 3.08 \mathrm{a}$ & $17.20 \pm 2.24 b$ & $16.75 \pm 2.61 \mathrm{e}$ & $16.50 \pm 0.59 \mathrm{e}$ & $23.00 \pm 1.93 b$ & $18.50 \pm 1.66 d$ & $29.47 \pm 2.35 \mathrm{a}$ & $21.50 \pm 4.39 c$ \\
\hline Histidine & $78.67 \pm 6.13 b$ & $85.17 \pm 6.18 \mathrm{a}$ & $101.50 \pm 2.20 a$ & $71.00 \pm 1.93 \mathrm{e}$ & $91.50 \pm 1.12 b$ & $77.50 \pm 1.92 d$ & $66.50 \pm 1.66 f$ & $83.50 \pm 1.95 c$ \\
\hline Glutamine & $30.51 \pm 3.35 b$ & $32.63 \pm 3.38 \mathrm{a}$ & $36.38 \pm 0.71 b$ & $39.85 \pm 0.85 a$ & $34.08 \pm 0.51 c$ & $28.10 \pm 0.61 \mathrm{e}$ & $19.50 \pm 0.59 f$ & $31.50 \pm 0.59 d$ \\
\hline Isoleucine & $25.98 \pm 4.30 \mathrm{a}$ & $24.61 \pm 4.31 b$ & $21.50 \pm 0.49 d$ & $40.00 \pm 0.35 a$ & $18.00 \pm 0.85 \mathrm{e}$ & $32.50 \pm 0.59 b$ & $23.25 \pm .22 c$ & $16.50 \pm 0.23 f$ \\
\hline Asparagine & $47.00 \pm 6.47 \mathrm{a}$ & $42.75 \pm 6.47 b$ & $33.50 \pm 0.59 e$ & $36.00 \pm 3.57 \mathrm{~d}$ & $44.50 \pm 1.12 c$ & $34.00 \pm 1.48 \mathrm{e}$ & $53.50 \pm 1.11 b$ & $67.75 \pm 1.55 \mathrm{a}$ \\
\hline Methionine & $17.08 \pm 2.22 \mathrm{a}$ & $22.13 \pm 6.62 \mathrm{a}$ & $12.63 \pm 2.14 \mathrm{a}$ & $11.00 \pm 0.35 \mathrm{a}$ & $13.63 \pm 0.35 a$ & $20.25 \pm 0.70 a$ & $20.13 \pm 1.59 a$ & $15.00 \pm 3.02 \mathrm{a}$ \\
\hline Serine & $106.08 \pm 8.13 a$ & $95.50 \pm 6.25 b$ & $78.00 \pm 1.83 f$ & $115.50 \pm 2.75 b$ & $90.75 \pm 2.10 \mathrm{e}$ & $104.50 \pm 2.75 c$ & $118.00 \pm 5.76 \mathrm{a}$ & $98.00 \pm 2.49 d$ \\
\hline Arginine & $23.63 \pm 4.29 b$ & $24.08 \pm 5.71 \mathrm{a}$ & $9.75 \pm 0.44 f$ & $29.50 \pm 0.59 c$ & $20.25 \pm 1.24 d$ & $15.50 \pm 0.59 f$ & $33.25 \pm 3.72 b$ & $34.88 \pm 0.27 a$ \\
\hline Proline & $94.67 \pm 6.90 \mathrm{a}$ & $103.42 \pm 7.52 \mathrm{a}$ & $118.75 \pm 2.90 \mathrm{a}$ & $87.25 \pm 2.09 \mathrm{e}$ & $111.25 \pm 2.63 b$ & $94.25 \pm 2.34 d$ & $78.00 \pm 1.93 f$ & $104.75 \pm 2.61 c$ \\
\hline Tyrosine & $38.75 \pm 4.16 \mathrm{a}$ & $27.25 \pm 5.16 b$ & $35.00 \pm 3.02 b$ & $31.25 \pm 3.18 d$ & $33.75 \pm 2.34 c$ & $26.50 \pm 2.75 f$ & $27.25 \pm 6.99 \mathrm{e}$ & $46.25 \pm 3.70 \mathrm{a}$ \\
\hline Glutamic acid & $159.50 \pm 10.15 b$ & $184.00 \pm 9.42 \mathrm{a}$ & $172.80 \pm 13.15 b$ & $168.75 \pm 10.00 b$ & $181.50 \pm 5.50 \mathrm{a}$ & $183.75 \pm 11.11 \mathrm{a}$ & $171.50 \pm 14.00 b$ & $152.25 \pm 11.91 \mathrm{C}$ \\
\hline Aspartic acid & $118.42 \pm 7.60 b$ & $103.83 \pm 6.53 \mathrm{a}$ & $89.00 \pm 3.06 f$ & $122.25 \pm 4.25 b$ & $98.00 \pm 3.57 \mathrm{e}$ & $117.25 \pm 4.25 c$ & $128.75 \pm 4.80 \mathrm{a}$ & $111.50 \pm 4.13 \mathrm{~d}$ \\
\hline Leucine & $30.42 \pm 5.44 a$ & $29.17 \pm 4.82 b$ & $29.75 \pm 5.35 c$ & $18.00 \pm 0.05 f$ & $28.75 \pm 1.28 d$ & $32.50 \pm 3.84 b$ & $45.25 \pm 1.55 \mathrm{a}$ & $24.50 \pm 3.30 \mathrm{e}$ \\
\hline Tryptophan & $7.45 \pm 2.25 \mathrm{a}$ & $5.83 \pm 2.17 b$ & $5.50 \pm 1.66 \mathrm{~d}$ & $2.88 \pm 1.58 \mathrm{e}$ & $9.50 \pm 1.12 b$ & $12.50 \pm 1.12 a$ & $1.50 \pm 0.82 f$ & $8.00 \pm 0.35 c$ \\
\hline
\end{tabular}

Values with different letters are significantly different

a Unit for amino acids: milligrams $(\mathrm{mg})$ per gram of protein

Significant differences were found for TC content between samples $(P<0.01)$, with the maximum and minimum TC contents recorded for the Kanisoor (45.56\%) and Sarvabad (39.83\%) regions, respectively. Interestingly, the female tree samples had higher TC contents than the males. Arabinose and galactose were the most abundant monosaccharides in the samples, suggesting an arabinogalactan structure for the gum [26]. It should be noted that the presence of fairly high concentrations of xylose and rhamnose may influence the physical properties $\left(T_{g}\right.$; Table 8) [27]. Substantial differences between samples from different regions $(P<0.01)$ could result from topographic and land attributes (slope, aspect, soil type, etc.) [28].

ANOVA results indicated that both geographic location $(P<0.01)$ and gender $(P<0.01)$ had significant effects on the amino acid concentrations (Table 2). Slope, geographical direction and such could influence these results [28]. The presence of high concentrations of serine and proline in all samples may be related to the arabinogalactan-protein content $[26,29]$.

Significant differences were observed for the volatile components ( $\mathrm{VoC}$ ) contents according to region
$(P<0.01)$ and gender $(P<0.01)$. In both female and male samples, $\alpha$-Pinene was determined to be the principle volatile component along with $\beta$-Pinene, longifolene, trans-verbenol, 3 -carene, comphene, $\alpha$-terpinolene and limonene (Table 3 ).

Måren [30] determined that concentrations of nitrogen, phosphorus, organic materials and moisture in the soil were high in the north and the level of soil acidity was low. These characterizations indicate that the efficiency of $\mathrm{VoC}$ from the samples taken in the north was higher than in the south. The samples from the south showed increased concentrations of $\mathrm{VoC}$ compared to samples from the north. Our results agree with these findings [30].

\section{Fourier transform infrared spectroscopy}

Chemical and covalent bonding characterization and detection of functional groups of PAK gum samples from the female and male trees of different regions was carried out using the transmittance spectra of the characteristic peaks in FTIR spectroscopy. Figure 1 shows the FTIR spectra of both female and male oleoresins. 
Table 3 Volatile components of the extracted gum samples for factors under investigation

\begin{tabular}{|c|c|c|c|c|c|c|c|c|c|}
\hline \multirow[t]{2}{*}{ Compound } & \multirow[t]{2}{*}{ RI } & \multicolumn{2}{|l|}{ Gender } & \multicolumn{6}{|c|}{ Geographic regions } \\
\hline & & Female & Male & Kanisoor & Armardeh & Hawraman & Dezli & Sarvabad & Marivan \\
\hline 1,3 Octanal & 873 & $1.15 \pm 0.16 a$ & $0.91 \pm 0.25 b$ & $1.25 \pm 0.22 b$ & $1.36 \pm 0.03 a$ & $1.00 \pm 0.16 c$ & $0.43 \pm 0.23 e$ & $1.25 \pm 0.14 b$ & $0.90 \pm 0.08 d$ \\
\hline Tricyclene & 898 & $0.98 \pm 0.08 b$ & $1.06 \pm 0.09 a$ & $1.00 \pm 0.04 \mathrm{a}$ & $0.85 \pm 0.02 b$ & $1.00 \pm 0.03 \mathrm{a}$ & $1.10 \pm 0.03 \mathrm{a}$ & $1.10 \pm 0.07 a$ & $1.08 \pm 0.17 a$ \\
\hline a-Thujene & 908 & $1.64 \pm 0.16 a$ & $1.60 \pm 0.25 b$ & $1.70 \pm 0.03 b$ & $1.70 \pm 0.19 b$ & $1.95 \pm 0.11 \mathrm{a}$ & $1.35 \pm 0.22 c$ & $1.95 \pm 0.13 a$ & $1.08 \pm 0.04 d$ \\
\hline a-Pinene & 920 & $44.41 \pm 2.16 \mathrm{a}$ & $43.39 \pm 1.93 b$ & $40.15 \pm 1.07 \mathrm{e}$ & $41.00 \pm 1.69 d$ & $45.05 \pm 2.00 \mathrm{~b}$ & $44.80 \pm 0.14 b$ & $42.83 \pm 1.63 c$ & $49.58 \pm 1.26 a$ \\
\hline Comphene & 935 & $2.63 \pm 0.73 b$ & $3.32 \pm 0.93 a$ & $3.48 \pm 0.09 b$ & $3.10 \pm 0.95 b$ & $3.05 \pm 0.82 c$ & $3.05 \pm 0.90 c$ & $1.43 \pm 0.09 c$ & $3.83 \pm 1.51 \mathrm{a}$ \\
\hline verbenone & 946 & $1.20 \pm 0.17 b$ & $0.88 \pm 0.35 a$ & $0.50 \pm 0.27 \mathrm{e}$ & $0.96 \pm 0.05 c$ & $1.20 \pm 0.05 b$ & $1.78 \pm 0.07 a$ & $0.78 \pm 0.43 d$ & $1.00 \pm 0.04 c$ \\
\hline Sabinene & 960 & $1.53 \pm 0.22 \mathrm{a}$ & $1.35 \pm 0.21 b$ & $1.20 \pm 0.19 c$ & $2.10 \pm 0.03 a$ & $1.18 \pm 0.07 c$ & $1.20 \pm 0.18 c$ & $1.73 \pm 0.15 b$ & $1.25 \pm 0.08 c$ \\
\hline$\beta$-Pinene & 971 & $4.41 \pm 0.42 b$ & $5.55 \pm 0.74 \mathrm{a}$ & $5.20 \pm 0.08 c$ & $6.10 \pm 0.74 a$ & $4.65 \pm 0.05 d$ & $4.65 \pm 0.08 d$ & $5.55 \pm 0.65 b$ & $3.73 \pm 0.55 \mathrm{e}$ \\
\hline Myrcene & 990 & $1.57 \pm 0.18 a$ & $1.58 \pm 0.25 a$ & $1.75 \pm 0.18 \mathrm{ab}$ & $1.30 \pm 0.19 d$ & $1.83 \pm 0.21 \mathrm{a}$ & $1.63 \pm 0.14 b c$ & $1.48 \pm 0.24 c$ & $1.48 \pm 0.28 c$ \\
\hline 3-Carene & 1006 & $3.40 \pm 0.63 \mathrm{a}$ & $1.89 \pm 0.82 b$ & $1.98 \pm 0.53 \mathrm{~cd}$ & $1.93 \pm 1.06 \mathrm{~d}$ & $2.53 \pm 1.38 b c$ & $2.68 \pm 0.48 b$ & $4.45 \pm 0.04 \mathrm{a}$ & $2.33 \pm 0.15 \mathrm{bcd}$ \\
\hline a-Phelandrene & 1020 & $1.50 \pm 0.19 b$ & $1.71 \pm 0.29 a$ & $2.23 \pm 0.15 a$ & $1.68 \pm 0.16 c$ & $0.95 \pm 0.02 \mathrm{e}$ & $1.28 \pm 0.12 d$ & $1.85 \pm 0.08 b$ & $1.65 \pm 0.25 c$ \\
\hline$\beta$-Ocimene & 1031 & $1.49 \pm 0.23 \mathrm{a}$ & $1.13 \pm 0.13 b$ & $1.45 \pm 0.22 b$ & $1.04 \pm 0.08 d$ & $1.48 \pm 0.32 b$ & $1.28 \pm 0.15 c$ & $1.63 \pm 0.14 \mathrm{a}$ & $1.00 \pm 0.02 d$ \\
\hline p-Cymen & 1040 & $1.41 \pm 0.17 b$ & $1.60 \pm 0.26 a$ & $1.53 \pm 0.07 b$ & $1.03 \pm 0.02 \mathrm{e}$ & $1.93 \pm 0.26 a$ & $1.40 \pm 0.25 c$ & $1.25 \pm 0.14 d$ & $1.90 \pm 0.08 \mathrm{a}$ \\
\hline Fenchone & 1052 & $0.73 \pm 0.28 b$ & $1.35 \pm 0.20 a$ & $0.38 \pm 0.20 \mathrm{e}$ & $1.18 \pm 0.13 b c$ & $1.08 \pm 0.02 \mathrm{~cd}$ & $1.20 \pm 0.16 b$ & $1.00 \pm 0.55 d$ & $1.43 \pm 0.04 a$ \\
\hline Limonene & 1065 & $2.85 \pm 0.47 b$ & $3.96 \pm 0.84 \mathrm{a}$ & $3.30 \pm 0.41 d$ & $4.65 \pm 0.80 a$ & $3.30 \pm 0.14 d$ & $3.80 \pm 0.08 c$ & $4.40 \pm 0.52 b$ & $0.98 \pm 0.02 \mathrm{e}$ \\
\hline 1,8-Cineole & 1084 & $1.08 \pm 0.09 b$ & $1.66 \pm 0.23 a$ & $1.53 \pm 0.26 b$ & $1.00 \pm 0.08 d$ & $1.54 \pm 0.32 b$ & $1.45 \pm 0.08 c$ & $0.99 \pm 0.01 \mathrm{~d}$ & $1.72 \pm 0.21 \mathrm{a}$ \\
\hline Y-Terpinene & 1105 & $1.53 \pm 0.23 a$ & $1.21 \pm 0.13 b$ & $1.00 \pm 0.04 c$ & $1.75 \pm 0.16 a$ & $1.44 \pm 0.06 b$ & $1.70 \pm 0.19 a$ & $1.36 \pm 0.23 b$ & $0.98 \pm 0.02 c$ \\
\hline a-Terpinolene & 1122 & $2.73 \pm 0.71 b$ & $3.01 \pm 0.55 \mathrm{a}$ & $2.13 \pm 0.02 c$ & $4.81 \pm 0.04 \mathrm{a}$ & $2.00 \pm 0.33 c$ & $3.20 \pm 0.65 b$ & $1.84 \pm 0.26 \mathrm{~d}$ & $3.24 \pm 0.54 b$ \\
\hline Citronellel & 1140 & $1.32 \pm 0.38 b$ & $1.35 \pm 0.23 a$ & $1.95 \pm 0.06 \mathrm{a}$ & $1.63 \pm 0.37 b$ & $1.40 \pm 0.19 c$ & $1.35 \pm 0.16 c$ & $0.90 \pm 0.04 d$ & $0.78 \pm 0.43 e$ \\
\hline Linalool & 1162 & $1.43 \pm 0.23 a$ & $1.35 \pm 0.24 b$ & $1.60 \pm 0.07 b$ & $1.00 \pm 0.08 \mathrm{e}$ & $1.25 \pm 0.20 c$ & $2.20 \pm 0.04 \mathrm{a}$ & $1.14 \pm 0.02 d$ & $1.15 \pm 0.16 \mathrm{~cd}$ \\
\hline Cis-verbenol & 1180 & $0.80 \pm 0.34 b$ & $0.89 \pm 0.24 \mathrm{a}$ & $1.00 \pm 0.04 c$ & $0.75 \pm 0.41 d$ & $0.48 \pm 0.26 \mathrm{e}$ & $0.38 \pm 0.20 f$ & $1.30 \pm 0.33 a$ & $1.18 \pm 0.05 b$ \\
\hline $\begin{array}{l}\text { Trans-pinocar- } \\
\text { veol }\end{array}$ & 1196 & $1.03 \pm 0.23 b$ & $1.18 \pm 0.14 \mathrm{a}$ & $1.58 \pm 0.05 a$ & $0.93 \pm 0.10 \mathrm{~d}$ & $1.08 \pm 0.14 c$ & $1.13 \pm 0.11 c$ & $0.50 \pm 0.29 \mathrm{e}$ & $1.40 \pm 0.14 b$ \\
\hline Trans-verbenol & 1212 & $4.95 \pm 0.61 b$ & $5.13 \pm 0.70 a$ & $5.85 \pm 0.86 a$ & $5.93 \pm 0.56 a$ & $4.15 \pm 0.30 c$ & $4.63 \pm 0.37 b$ & $5.73 \pm 0.57 a$ & $3.95 \pm 0.05 c$ \\
\hline Terpinen-4-ol & 1240 & $1.17 \pm 0.11 \mathrm{a}$ & $1.03 \pm 0.26 b$ & $1.13 \pm 0.10 b$ & $1.55 \pm 0.07 a$ & $1.13 \pm 0.05 b$ & $1.15 \pm 0.11 b$ & $1.18 \pm 0.07 b$ & $0.48 \pm 0.26 c$ \\
\hline Myrtenal & 1264 & $1.08 \pm 0.11 b$ & $1.23 \pm 0.12 \mathrm{a}$ & $1.50 \pm 0.08 \mathrm{a}$ & $0.93 \pm 0.05 d$ & $1.15 \pm 0.11 \mathrm{~b}$ & $1.13 \pm 0.14 b c$ & $1.13 \pm 0.07 b c$ & $1.08 \pm 0.02 c$ \\
\hline Myrtenol & 1277 & $1.11 \pm 0.14 \mathrm{a}$ & $0.84 \pm 0.22 b$ & $1.11 \pm 0.19 b$ & $1.34 \pm 0.06 a$ & $0.83 \pm 0.01 \mathrm{~d}$ & $1.04 \pm 0.05 c$ & $0.66 \pm 0.36 d$ & $0.88 \pm 0.08 d$ \\
\hline Trans-carveol & 1297 & $0.92 \pm 0.23 b$ & $1.25 \pm 0.19 a$ & $1.20 \pm 0.08 b$ & $1.10 \pm 0.08 c$ & $1.23 \pm 0.05 b$ & $0.53 \pm 0.23 \mathrm{e}$ & $0.84 \pm 0.02 d$ & $1.63 \pm 0.19 a$ \\
\hline Bornyl acetate & 1339 & $1.15 \pm 0.12 \mathrm{a}$ & $0.71 \pm 0.26 b$ & $1.20 \pm 0.08 \mathrm{a}$ & $1.06 \pm 0.06 b$ & $1.25 \pm 0.17 a$ & $0.48 \pm 0.26 d$ & $1.04 \pm 0.05 b$ & $0.56 \pm 0.30 c$ \\
\hline p-Cymen-7-ol & 1355 & $0.83 \pm 0.21 b$ & $0.94 \pm 0.21 \mathrm{a}$ & $0.90 \pm 0.02 c$ & $0.00 \mathrm{~d}$ & $0.93 \pm 0.11 \mathrm{c}$ & $1.27 \pm 0.02 \mathrm{a}$ & $1.00 \pm 0.14 b$ & $1.24 \pm 0.17 a$ \\
\hline a-Burbonene & 1369 & $0.94 \pm 0.10 b$ & $1.13 \pm 0.11 \mathrm{a}$ & $0.85 \pm 0.02 d$ & $1.05 \pm 0.11 b$ & $1.14 \pm 0.10 \mathrm{a}$ & $1.19 \pm 0.14 \mathrm{a}$ & $1.04 \pm 0.15 b$ & $0.95 \pm 0.02 c$ \\
\hline $\begin{array}{l}\text { a-Terpenyl } \\
\text { acetate }\end{array}$ & 1396 & $1.29 \pm 0.12 \mathrm{a}$ & $1.09 \pm 0.12 b$ & $1.43 \pm 0.04 \mathrm{a}$ & $1.29 \pm 0.18 b$ & $1.18 \pm 0.13 c$ & $1.05 \pm 0.02 \mathrm{~d}$ & $1.14 \pm 0.16 \mathrm{~cd}$ & $1.06 \pm 0.11 d$ \\
\hline Longifolene & 1440 & $3.91 \pm 0.71 \mathrm{a}$ & $3.23 \pm 1.02 b$ & $5.30 \pm 0.41 \mathrm{a}$ & $2.35 \pm 0.65 f$ & $2.70 \pm 0.48 \mathrm{e}$ & $3.93 \pm 0.37 c$ & $4.34 \pm 0.63 b$ & $2.83 \pm 1.00 \mathrm{~d}$ \\
\hline Germacren D & 1505 & $0.96 \pm 0.11 \mathrm{a}$ & $0.88 \pm 0.22 b$ & $0.91 \pm 0.02 \mathrm{~d}$ & $0.80 \pm 0.07 e$ & $0.40 \pm 0.22 f$ & $1.10 \pm 0.03 b$ & $0.98 \pm 0.02 c$ & $1.35 \pm 0.02 a$ \\
\hline a-Muurolene & 1537 & $0.64 \pm 0.25 a$ & $0.61 \pm 0.25 b$ & $0.38 \pm 0.20 \mathrm{e}$ & $0.92 \pm 0.16 b$ & $1.01 \pm 0.16 a$ & $0.49 \pm 0.26 d$ & $0.36 \pm 0.14 \mathrm{e}$ & $0.58 \pm 0.31 c$ \\
\hline $\begin{array}{l}\text { Total volatile } \\
\text { components } \\
(\%)\end{array}$ & & 27.88 & 27.39 & 26.47 & 26.73 & 28.67 & 27.04 & 28.28 & 28.59 \\
\hline
\end{tabular}

Values with different letters are significantly different

The results of FTIR show that the pattern of the fingerprint region $\left(500-1500 \mathrm{~cm}^{-1}\right)$ is similar for nearly all gum samples. This spectrum causes complex deformation of transmittance, which is unique for each compound, and this range cannot be used for recognition of uncertain components [31]. In the PAK spectrum, the band range at $4000-3750 \mathrm{~cm}^{-1}$ represents the existence of water vapor [32]. The characteristic transmission band at 3500$3200 \mathrm{~cm}^{-1}$ relates to the $\mathrm{O}-\mathrm{H}$ stretch vibration, strong dimer $\mathrm{O}-\mathrm{H}$ stretching vibration, $\mathrm{N}-\mathrm{H}$ stretching vibration and aromatic secondary $\left(2^{\circ}\right)$ amine [33]. The results of FTIR spectra for nearly all PAK gum samples indicate a band at $3000-2900 \mathrm{~cm}^{-1}$ that was assigned to aliphatic $\mathrm{C}-\mathrm{H}$ stretching related to the existence of galactose, 

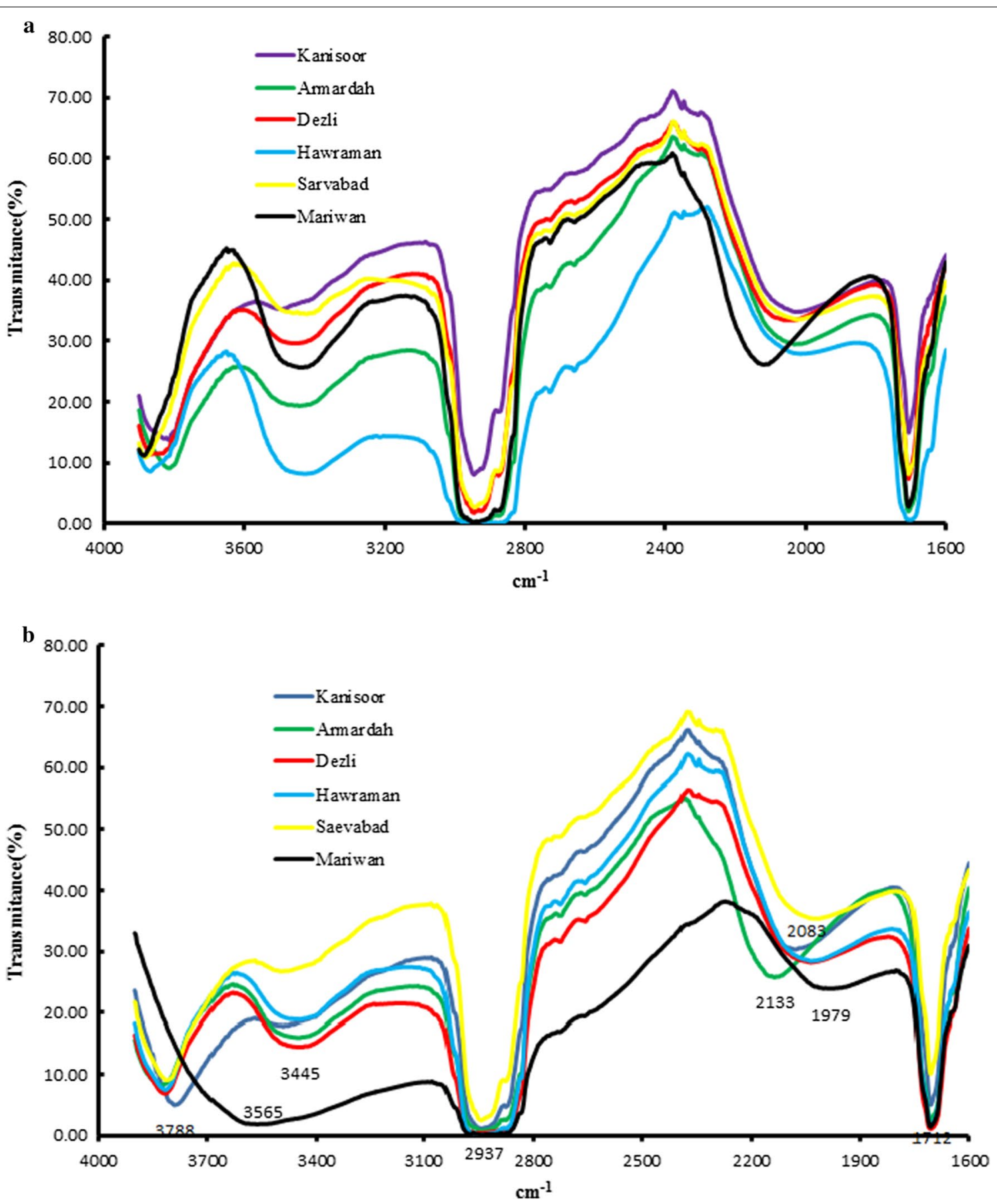

Fig. 1 FTIR spectra of kurdica gum samples from the female (a) and the male (b) trees of different regions in Kurdistan province, Iran

arabinose and rhamnose [34]. The spectra of nearly all PAK samples showed a band at $2150-1900 \mathrm{~cm}^{-1}$ can be assigned to aromatic cyanide and cyanate. The spectra of nearly all PAK gum samples showed a band at 1725$1700 \mathrm{~cm}^{-1}$ which can be assigned to the presence of carboxylic acid stretching and ketone [35]. FTIR results for female samples of the content and intensity of functional groups were higher than for the male samples in all regions. 


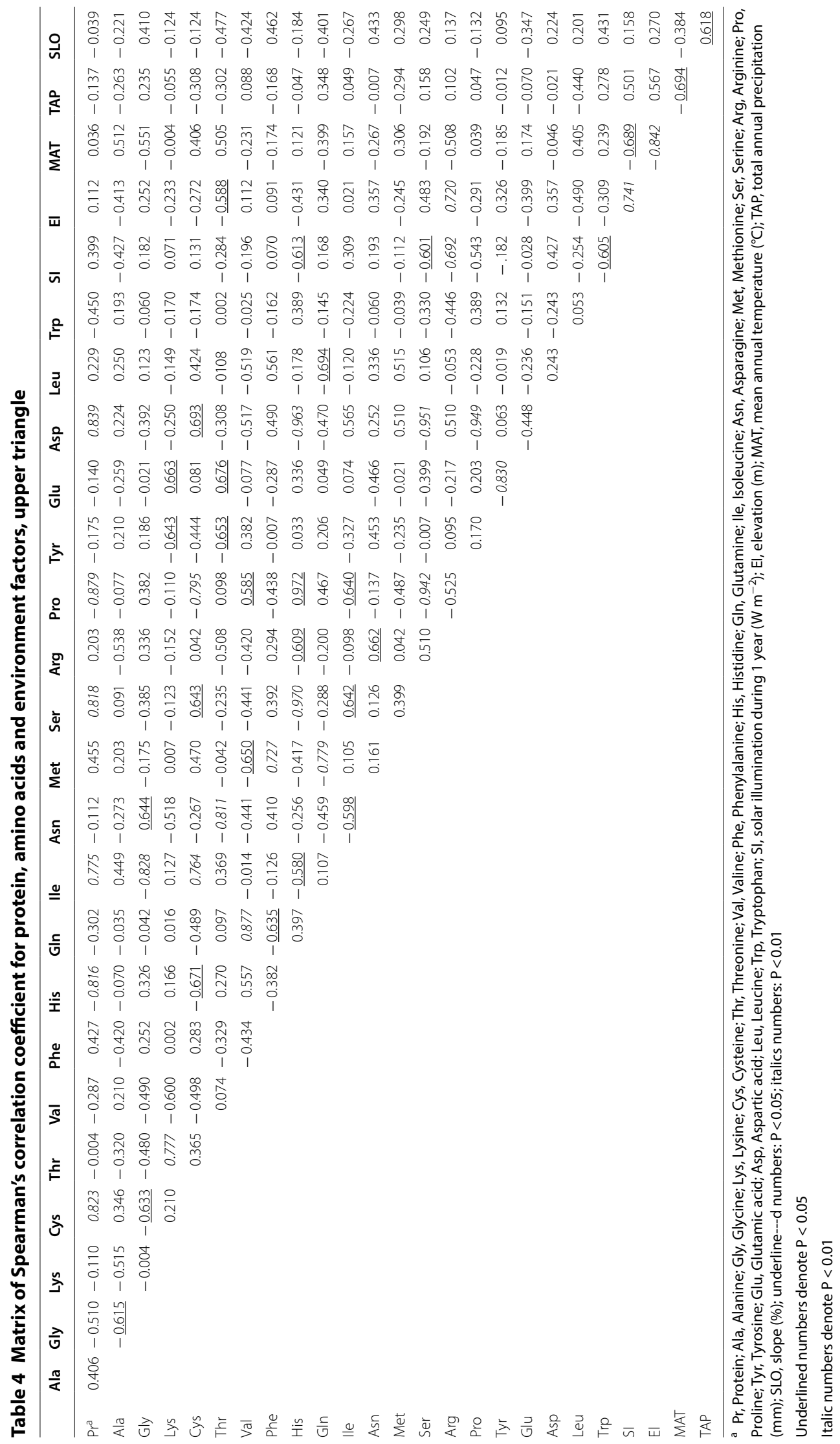




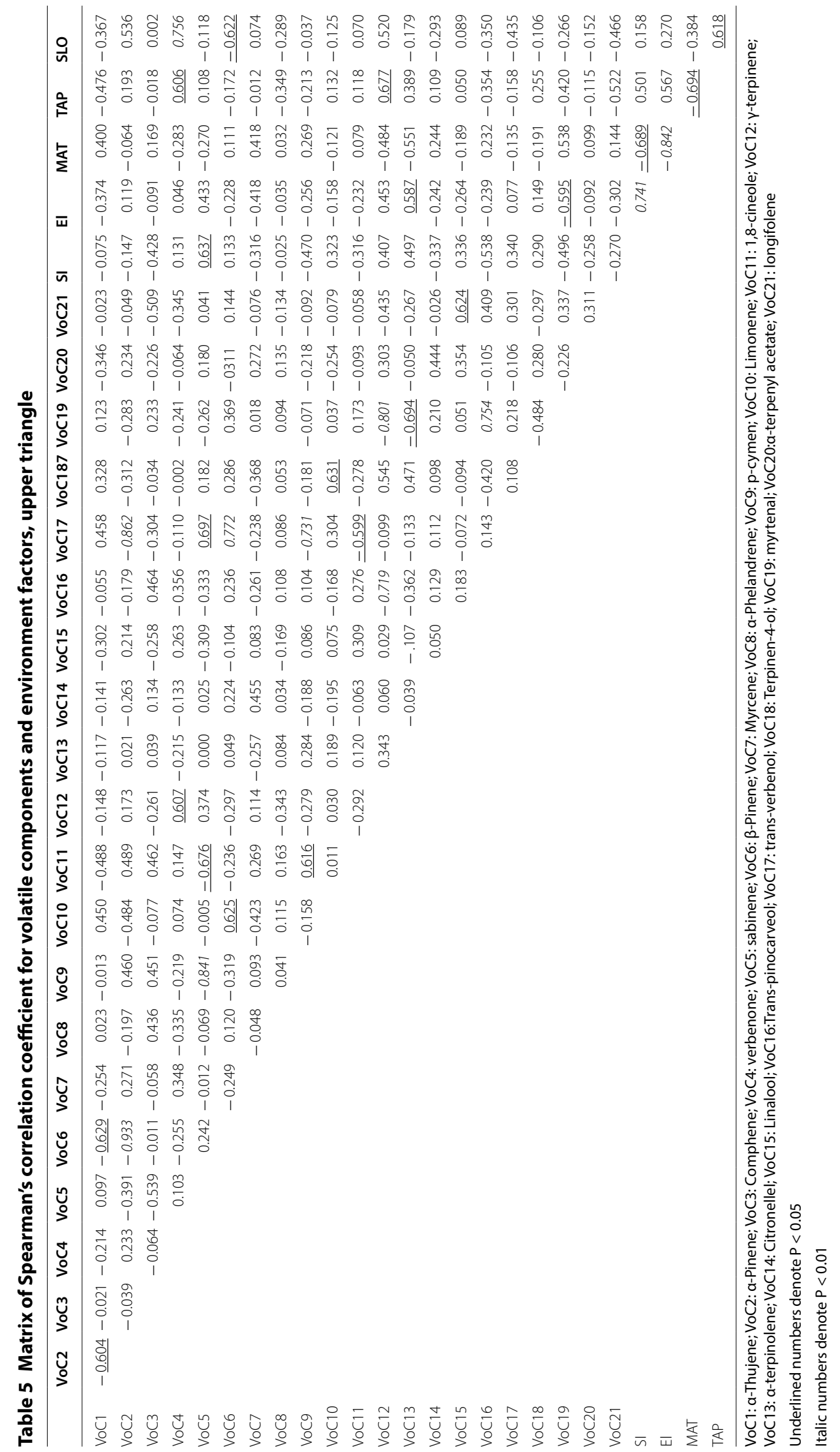


Table 6 Matrix of Spearman's correlation coefficient for ash, moisture, carbohydrates and environment factors, upper triangle

\begin{tabular}{|c|c|c|c|c|c|c|c|c|c|c|c|c|c|c|c|}
\hline & Ash & TS & Ara & Gal & Rha & Fuc & Xyl & Glc & GlcA & GalA & SI & El & MAT & TAP & SLO \\
\hline $\mathrm{MO}$ & -0.175 & 0.287 & 0.476 & -0.070 & -0.126 & -0.462 & -0.382 & -0.021 & -0.218 & 0.165 & -0.133 & 0.224 & -0.298 & 0.021 & -0.298 \\
\hline Ash & & -0.196 & -0.102 & 0.161 & 0.280 & 0.081 & 0.239 & 0.273 & 0.295 & -0.042 & -0.441 & -0.517 & 0.494 & -0.469 & -0.207 \\
\hline TS & & & 0.308 & -0.105 & -0.098 & -0.420 & -0.046 & -0.091 & -0.710 & -0.217 & -0.371 & -0.357 & 0.270 & -0.343 & -0.497 \\
\hline Ara & & & & $\underline{0.599}$ & -0.708 & -0.912 & -0.898 & -0.648 & -0.306 & 0.488 & -0.259 & 0.312 & -0.025 & -0.139 & -0.358 \\
\hline Gal & & & & & -0.839 & -0.676 & -0.754 & -0.804 & 0.018 & 0.347 & 0.028 & 0.217 & 0.188 & -0.039 & -0.403 \\
\hline Rha & & & & & & 0.781 & 0.881 & 0.916 & 0.309 & -0.455 & -0.224 & -0.455 & 0.028 & -0.074 & 0.403 \\
\hline Fuc & & & & & & & 0.852 & $\underline{0.630}$ & 0.403 & -0.386 & 0.130 & -0.242 & -0.021 & 0.207 & $\underline{0.600}$ \\
\hline Xyl & & & & & & & & 0.775 & 0.183 & -0.543 & -0.007 & -0.502 & 0.125 & -0.012 & 0.322 \\
\hline Glc & & & & & & & & & 0.211 & -0.294 & -0.112 & -0.378 & -0.014 & -0.263 & 0.175 \\
\hline GlcA & & & & & & & & & & -0.239 & -0.144 & -0.095 & 0.063 & 0.276 & $\underline{0.590}$ \\
\hline GalA & & & & & & & & & & & 0.350 & $\underline{0.630}$ & -0.247 & -0.193 & -0.102 \\
\hline SI & & & & & & & & & & & & 0.741 & -0.689 & 0.501 & 0.158 \\
\hline $\mathrm{El}^{\mathrm{a}}$ & & & & & & & & & & & & & -0.842 & 0.567 & 0.270 \\
\hline MAT & & & & & & & & & & & & & & -0.694 & -0.384 \\
\hline TAP & & & & & & & & & & & & & & & $\underline{0.618}$ \\
\hline
\end{tabular}

MO, moisture; TS, total sugar; Ara, arabinose; Gal, galactose; Rha, rhamnose; Fuc, fucose; Xyl, xylose; Glc, glucose; GlcA, glucuronic acid; GalA, galacturonic acid Underlined numbers denote $\mathrm{P}<0.05$

Italic numbers denote $\mathrm{P}<0.01$

\section{Statistical relationships}

Spearman rank correlation coefficient results among oleoresin properties and environment factors (slope, annual solar illumination, elevation, mean annual temperature and total annual precipitation) are shown in Tables 4, 5 and 6. Fucose, glucuronic acid and $\beta$-pinene $(P<0.05)$ and verbenone $(P<0.01)$ contents display significant positive correlation with terrain slope. Solar illumination showed significantly negative correlations with histidine and serine at $P<0.05(\mathrm{r}=(-0.613)-(-0.558))$ and with arginine at $P<0.01(\mathrm{r}=-0.692)$. Except for sabinene, no significant correlation observed between volatile components and solar illumination. Elevation significantly correlates with arginine at $P<0.01(\mathrm{r}=0.720)$ and with galacturonic acid and $\alpha$-terpinolene at $P<0.05(\mathrm{r}=0.630$ and 0.587 , respectively). Also, elevation is found to be negatively correlated with threonine $(r=-0.588)$. The significant correlation between chemical components and total annual precipitation was observed only for verbenone and $\alpha$-terpinene at $P<0.05(\mathrm{r}=0.606$ and 0.677 , respectively).

Previous studies have showed clear evidence that amino acid compositions have high correlation with environment characteristics (solar illumination, elevation, mean annual temperature and total annual precipitation) [36-42]. It is well-established in the literature that environmental factors (solar illumination, total annual precipitation) plays a fundamental role in the control content of carbohydrates production by photosynthesis
[43-47]. According to Maxwell [49], changes in climate will strongly affect environmental factors. These changes in turn will affect availability of important resources for plants such as degree days, light, soil moisture, soil nutrients and soil oxygen that will potentially result in large alterations, both qualitatively and quantitatively, in production of secondary compounds [39, 48-51].

\section{Thermogravimetric analysis}

TGA is a simple and precise method used to assay the decomposition model and the thermal stability of polymers. The results of TGA and derivative mass loss measurements of PAK gum samples are shown in Table 7.

The primary and derivative thermograms for the PAK gum samples in the TGA curves showing the details of thermal behavior are given in Table 7. Nearly all samples of the various regions exhibited double major stage decomposition patterns, except for the female trees of the Dezli and Hawraman regions (which have three stages). The first stage occurs at $80{ }^{\circ} \mathrm{C}$ and can be attributed to the desorption of volatile essential oils and the loss of moisture and structural water, which in turn is associated with the hydrochloric nature of the functional groups of the PAK gum samples (mass loss of $20 \%$ to $25 \%$ ) [52].

The second stage of the samples from Dezli and Hawraman occurred at 200 to $300{ }^{\circ} \mathrm{C}$ (mass loss of $45 \%$ to $53 \%$ ) and the main decomposition of samples started above 240 to $360^{\circ} \mathrm{C}$ (mass loss of $80 \%$ to $92 \%$ ), both due to polysaccharide and polypeptide thermal decomposition [12, 
Table 7 Thermo-gravimetric data of PAK gum samples from the female and the male trees of different regions in Kurdistan province, Iran

\begin{tabular}{|c|c|c|c|c|c|c|}
\hline Gender & Regions & $\begin{array}{l}\text { No. of decomposition } \\
\text { stage }\end{array}$ & Temprature range $\left({ }^{\circ} \mathrm{C}\right)$ & $\begin{array}{l}\text { Derivative mass loss } \\
\text { peak }\left({ }^{\circ} \mathrm{C}\right)\end{array}$ & Mass loss $\%$ & $\begin{array}{l}\text { Activation energy } \\
\left(\mathrm{kJ} \mathrm{mol}^{-1}\right)^{\mathrm{a}}\end{array}$ \\
\hline \multirow[t]{26}{*}{ Female } & Armardeh & 1 & $27.11-162$ & 93.68 & 22.53 & 52.24 \\
\hline & & 2 & $162-275$ & - & 32.60 & - \\
\hline & & 3 & $275-402$ & 362.3 & 91.15 & 56.42 \\
\hline & & 4 & $402-600$ & - & 99.54 & - \\
\hline & Kanisur & 1 & $30.71-149$ & 86.47 & 21.96 & 55.96 \\
\hline & & 2 & $149-241$ & - & 29.75 & - \\
\hline & & 3 & $241-379$ & 339.80 & 80.02 & 39.77 \\
\hline & & 4 & $379-600$ & 405.76 & 99.48 & 47.81 \\
\hline & Dezli & 1 & $26.79-164.82$ & 77.21 & 24.86 & 47.64 \\
\hline & & 2 & $165.133-207.31$ & - & 28.84 & - \\
\hline & & 3 & $207.70-286.67$ & 257.99 & 43.39 & 14.94 \\
\hline & & 4 & $287.22-399.33$ & 359.46 & 92.19 & 50.96 \\
\hline & & 5 & $399.43-600$ & - & 99.5 & - \\
\hline & Hawraman & 1 & $29.60-142.74$ & 84.07 & 19.82 & 68.92 \\
\hline & & 2 & $143.20-201.06$ & - & 25.99 & - \\
\hline & & 3 & 201.83-309.75 & 289.67 & 52.65 & 28.08 \\
\hline & & 4 & $310.40-388.08$ & 352.17 & 90.52 & 48.98 \\
\hline & & 5 & $388.89-600$ & - & 99.52 & - \\
\hline & Sarvabad & 1 & $29.67-149.85$ & 93.07 & 19.05 & 66.98 \\
\hline & & 2 & $150.21-278.82$ & - & 33.91 & - \\
\hline & & 3 & $279.17-402.36$ & 354.315 & 90.34 & 52.28 \\
\hline & & 4 & $402.36-600$ & - & 99.71 & - \\
\hline & Marivan & 1 & $29.80-160.92$ & 80.28 & 22.42 & 64.06 \\
\hline & & 2 & $161.26-276.08$ & - & 32.87 & - \\
\hline & & 3 & $276.12-402.73$ & 357.59 & 90.22 & 52.78 \\
\hline & & 4 & $403-600$ & - & 99.52 & - \\
\hline \multirow[t]{24}{*}{ Male } & Armardeh & 1 & $26.67-156.23$ & 84.20 & 23.43 & 62.51 \\
\hline & & 2 & $157.19-241.49$ & - & 29.49 & - \\
\hline & & 3 & $242.638-409.53$ & 344.14 & 87.10 & 39.62 \\
\hline & & 4 & $410.18-600$ & - & 99.51 & - \\
\hline & Kanisur & 1 & $26.49-171.38$ & 85.20 & 23.46 & 58.93 \\
\hline & & 2 & $172.273-256.47$ & - & 29.49 & - \\
\hline & & 3 & $320.131-395.24$ & 350.73 & 87.1 & 48.83 \\
\hline & & 4 & $395.64-600$ & - & 99.51 & - \\
\hline & Dezli & 1 & $26.79-164.82$ & 80 & 27.72 & 45.22 \\
\hline & & 2 & $165.133-207.31$ & - & 51.48 & - \\
\hline & & 3 & $207.70-286.67$ & 270.69 & 91.18 & 28.5 \\
\hline & & 4 & 287.22-399.33 & 349.85 & 99.62 & 54 \\
\hline & Hawraman & 1 & $31.38-124.21$ & 93.15 & 14.07 & 53.02 \\
\hline & & 2 & $124.66-292.79$ & - & 33.55 & - \\
\hline & & 3 & $292.80-401.55$ & 363.23 & 90.97 & 57.97 \\
\hline & & 4 & $402.315-600$ & - & 99.71 & - \\
\hline & Sarvabad & 1 & $32.60-179.97$ & 97.65 & 23.25 & 59.22 \\
\hline & & 2 & $180.36-273.40$ & - & 30.75 & - \\
\hline & & 3 & $273.56-402.68$ & 358.69 & 90.09 & 57.59 \\
\hline & & 4 & $402.98-600$ & - & 99.52 & - \\
\hline & Marivan & 1 & $26.86-197.66$ & 83.30 & 28.15 & 64.06 \\
\hline & & 2 & $198.40-309.77$ & 278.21 & 56.12 & - \\
\hline & & 3 & 309.83-385.38 & 350.73 & 90 & 52.78 \\
\hline & & 4 & $385.68-600$ & - & 99.57 & - \\
\hline
\end{tabular}

\footnotetext{
${ }^{a}$ Activation energy for the major stage of decomposition according to the Borido method
} 
Table 8 DSC thermo-gram data of PAK gum samples from the female and the male trees of different regions in Kurdistan province, Iran

\begin{tabular}{|c|c|c|c|c|c|c|c|c|}
\hline \multirow[t]{2}{*}{ Regions } & \multirow[t]{2}{*}{ Gender } & \multicolumn{2}{|c|}{$\begin{array}{l}\text { Glass transition } \\
\text { temperature }\left(T_{g} 1\right)\end{array}$} & \multicolumn{2}{|c|}{$\begin{array}{l}\text { Glass transition } \\
\text { temperature }\left(T_{g} 2\right)\end{array}$} & \multirow{2}{*}{$\begin{array}{l}\text { Peak of melting } \\
\text { temperature }\left(T_{m}\right) \\
\left({ }^{\circ} \mathrm{C}\right)\end{array}$} & \multirow{2}{*}{$\begin{array}{l}\text { Enthalpy } \\
\text { changes (DH) } \\
\left(\mathrm{J} \mathrm{g}^{-1}\right)\end{array}$} & \multirow{2}{*}{$\begin{array}{l}\text { Heat capacity } \\
{\left[C_{p}\left(\mathrm{~J} \mathrm{~g}^{-1} \mathrm{k}^{-1}\right]\right.} \\
15\left({ }^{\circ} \mathrm{C}\right)\end{array}$} \\
\hline & & $\mathrm{T}_{\mathrm{g}}\left({ }^{\circ} \mathrm{C}\right)$ & $\Delta C_{p}\left(\mathrm{~J} \mathrm{~g}^{-1} \mathrm{k}^{-1}\right)$ & $\mathrm{T}_{\mathrm{g}}\left({ }^{\circ} \mathrm{C}\right)$ & $\Delta C_{p}\left(\mathrm{~J} \mathrm{~g}^{-1} \mathrm{k}^{-1}\right)$ & & & \\
\hline \multirow[t]{2}{*}{ Armardeh } & Female & -34.08 & 0.19 & 49.95 & 44.11 & - & - & 1.71 \\
\hline & Male & -29.21 & 0.16 & - & - & - & - & 1.78 \\
\hline \multirow[t]{2}{*}{ Kanisoor } & Female & -25.86 & 0.22 & 49.25 & 11.03 & - & - & 1.67 \\
\hline & Male & -24.95 & 0.08 & 65.18 & 46.59 & - & - & 1.77 \\
\hline \multirow[t]{2}{*}{ Dezli } & Female & -27.28 & 0.25 & 39.48 & 27.47 & 61.52 & -0.30 & 1.70 \\
\hline & Male & -31.43 & 0.23 & 72.27 & 81.00 & 51.19 & -0.24 & 1.74 \\
\hline \multirow[t]{2}{*}{ Hawraman } & Female & -27.49 & 0.24 & 46.24 & 3.43 & 63.85 & -0.17 & 1.68 \\
\hline & Male & -27.84 & 0.24 & 64.80 & 30.63 & - & - & 1.79 \\
\hline \multirow[t]{2}{*}{ Sarvabad } & Female & -24.16 & 0.21 & 49.81 & 6.37 & - & - & 1.72 \\
\hline & Male & -27.60 & 0.24 & 60.70 & 0.11 & - & - & 1.70 \\
\hline \multirow[t]{2}{*}{ Marivan } & Female & -26.15 & 0.263 & 42.07 & 33.01 & - & - & 1.71 \\
\hline & Male & -27.46 & 0.24 & 58.23 & 2.71 & - & - & 1.76 \\
\hline
\end{tabular}

52]. The peaks of the derivative mass loss curves of female trees occurred at 362.3, 339.80, 359.46, 352.17, 355.64 and $357.59{ }^{\circ} \mathrm{C}$ for Armardeh, Kanisoor, Dezli, Hawraman, Sarvabad and Marivan, respectively. The peaks of the derivative mass loss curves of the male trees appeared at 344.14, 350.73, 349.85, 363.23, 358.69 and 350.73 for Armardeh, Kanisoor, Dezli, Hawraman, Sarvabad and Marivan, respectively.

The stimulation of thermal decomposition can be quantitatively shown by determining the apparent activation energy $\left(E_{a}\right)$ for the main steps of decomposition of the samples. The Borido method is effective for the evaluation of the activation energy [53]. The Borido equation is:

$$
\ln \left(\ln \left(\frac{1}{y}\right)\right)=\ln \left(\ln \left(\frac{W_{0}-W_{\infty}}{W_{t}-W_{\infty}}\right)\right)=-\left(\frac{E_{a}}{R T}\right)+c
$$

where $W_{t}$ denotes the mass of the PAK gum samples at time $t$ and $W_{0}$ and $W_{\infty}$ denote the initial and final masses, respectively. From the slope of graphs of $\ln \left(\ln \left(\frac{1}{y}\right)\right)$ versus $\frac{1000}{T}$ ( $T$ denotes absolute temperature), the $E_{a}$ of any stage of decomposition could be evaluated $\left(\Upsilon=-6.0083 X+9.5883 ; R^{2}=0.9958\right)$. According to the data of the plots, the $E_{a}$ of the samples from female trees were lower than for male trees. In other words, the gum from female trees decomposed faster than from male trees (Table 7).

\section{Differential scanning calorimetry}

This method was used to determine the glass transition temperature, melting point, heat capacity and enthalpy of all PAK gum samples. The DSC thermograms of the PAK gum samples are shown in Table 8 along with the thermal transition behavior of the female and male gum samples. Nearly all gum samples had two glass transition temperatures, except for the male sample from the Armardeh region, which had only one $T_{g}$. The results showed that the $T_{g}$ for the male samples were higher than for the female samples. $T_{m}$ (Melting temperature) values occurred only for the Dezli and Hawraman regions and the exothermic enthalpy values for these samples showed an overall decrease in enthalpy by the generation of heat. The low glass transition temperature of all gum samples could relate to their low molecular weights signifying that glass to rubber transition occurs readily as a result of water sorption and can cause physical properties such as stickiness [54]. This is consistent with the apparent properties of the compound under consideration.

The DSC plots were also used to determine the heat capacity $\left(C_{P}\right)$ of the samples. Table 6 shows the heat capacity behavior of the female and male gum samples. As seen, the $C_{P}$ values of the female gum samples are lower than for the male samples, except for the samples from the Sarvabad region. The $C_{P}$ values at $15{ }^{\circ} \mathrm{C}$ show that they varied by region. Table 8 also shows that the male samples from the Hawraman region and the female samples from the Kanisoor region, recorded the maximum and minimum $C_{P}$ values, respectively.

The chemical composition and thermal properties of PAK gum samples determined from the female and male trees of different regions of Kurdistan province revealed that the female tree samples had higher TC contents than the male samples. Arabinose and galactose were the most abundant monosaccharides in the samples, suggesting an arabinogalactan structure of the analyzed gums. High concentrations of serine and proline may influence the arabinogalactan-proteins in the gum samples. In both the 
female and male samples, $\alpha$-Pinene was determined to be the principle volatile component. The PAK gum samples showed different thermal properties due to the functional group differences.

The TGA results indicated two-stage decomposition with the main decomposition of samples beginning above 240 to $360{ }^{\circ} \mathrm{C}$. The results of $E_{a}$ indicate that the female tree gums decomposed faster than the male tree gums and the minimum value of $E_{a}$ was observed for the samples collected from the Dezli region. The results of DSC indicated that the $T_{g}$ of nearly all the gum samples included two glass transition temperatures. The $T_{g}$ values of the male samples were higher than for the female samples. The $C_{P}$ value of all the female samples were lower than for the male samples. The maximum and minimum $C_{P}$ values were determined for samples from Hawraman and Kanisoor, respectively. The FTIR results showed that the content and intensity of the functional groups from the female samples were higher than for the male samples in all regions investigated. These results can improve our understanding about the optimization of PAK gum usage from different geographic regions.

\section{Authors' contribution}

FM, carried out gum sampling, spatial analyses, chemical and thermal analyses of gum samples, statistical analyses and drafted the manuscript. MM, carried out thermal analyses (DSC and TGA), interpreting obtained thermograms and participated in drafting the manuscript. RS, carried out FTIR and GC-Mass analyses and interpreting the obtained results. MHG, analysis of sugars and amino acids using HPLC and interpreting the obtained results. All authors read and approved the final manuscript.

\section{Author details}

1 Department of Food Science and Technology, Science and Research Branch, Islamic Azad University, Tehran, Iran. ${ }^{2}$ Department of Chemistry, University of Kurdistan, Sanandaj, Iran. ${ }^{3}$ Department of Chemistry, Science and Research Branch, Islamic Azad University, Tehran, Iran.

\section{Competing interest}

The authors declare that they have no competing interests.

\section{Publisher's Note}

Springer Nature remains neutral with regard to jurisdictional claims in published maps and institutional affiliations.

Received: 19 August 2018 Accepted: 21 January 2019

Published online: 22 February 2019

\section{References}

1. Zohary M (1952) A monographical study of the genus Pistacia. Palestine J Bot Jerus Ser 5:187-228

2. Martinez J-JI (2008) Impact of a gall-inducing aphid on Pistacia atlantica Desf. trees. Arthropod Plant Interact 2:147-151

3. Pazouki L, Mardi M, Shanjani PS, Hagidimitriou M, Pirseyedi SM, Naghavi MR, Avanzato D, Vendramin E, Kafkas S, Ghareyazie B, Ghaffari MR, Khayam Nekoui SM (2010) Genetic diversity and relationships among Pistacia species and cultivars. Conserv Genet 11:311-318

4. Farhoosh R, Tavakoli J, Khodaparast MHH (2008) Chemical composition and oxidative stability of kernel oils from two current subspecies of Pistacia atlantica in Iran. J Am Oil Chem Soc 85:723
5. Sharifi MS, Hazell SL (2011) GC-MS analysis and antimicrobial activity of the essential oil of the trunk exudates from Pistacia atlantica kurdica. J Pharm Sci Res 3:1364

6. Orhan I, Özçelik B, Aslan S, Kartal M, Karaoglu T, Şener B, Terzioglu S, Choudhary MI (2007) Antioxidant and antimicrobial actions of the clubmoss Lycopodium clavatum L. Phytochem Rev 6:189-196

7. Zhu B, Wang Q, Roge EF, Nan P, Liu Z, Zhong Y (2006) Chemical variation in leaf oils of Pistacia chinensis from five locations in China. Chem Nat Compd 42:422-425

8. Benhammou N, Bekkara FA, Panovska TK (2008) Antioxidant and antimicrobial activities of the Pistacia lentiscus and Pistacia atlantica extracts. Afr J Pharm Pharmacol 2:022-028

9. Rahimi AR, Hadyan J, Azizi M, Abdoosi V, Larijani K (2013) Comparison of chemical compounds found in the gum essential oil of male and female Pistacia atlantica subsp. Kurdica. J Biodivers Environ Sci 3:174-178

10. Barrero AF, Herrador MM, Arteaga JF, Akssira M, Mellouki F, Belgarrabe A, Blázquez MA (2005) Chemical composition of the essential oils of Pistacia atlantica Desf. J Essent Oil Res 17:52-54

11. Karamalla K (1999) Gum arabic production, chemistry and applications. University of Khartoum, Sudan, pp 4-30

12. Zohuriaan MJ, Shokrolahi F (2004) Thermal studies on natural and modified gums. Polym Test 23:575-579

13. Bothara SB, Singh S (2012) Thermal studies on natural polysaccharide. Asian Pac JTrop Biomed 2:S1031-S1035

14. Roos YH, Drusch S (2005) Phase transitions in foods. Academic Press, Cambridge

15. Sharifi MS (2006) Fractionations and analysis of trunk exudates from Pistacia genus in relation to antimicrobial activity. A thesis of Docotor of Philosophy, College of Health and Science, University of Western Sydney. Sydney, Australia

16. Karamshahi A, Tahmasbi M, Najafifar A (2005) Study the best method of resin extraction from Pistacia atlantica trees. Paj Saz 66:78-82 (In Persian)

17. Thiex N (2008) Evaluation of analytical methods for the determination of moisture, crude protein, crude fat, and crude fiber in distillers dried grains with solubles. J AOAC Int 92:61-73

18. Miller GL (1959) Protein determination of large numbers of samples. Anal Chem 31:964

19. Rasouli Z, Akbari-Adergani B (2016) Assessment of aspartame exposure due to consumption of some imported chewing gums by microwave digestion and high performance liquid chromatography analysis. Orient J Chem 32:1649-1658

20. Masuko T, Minami A, Iwasaki N, Majima T, Nishimura S-I, Lee YC (2005) Carbohydrate analysis by a phenol-sulfuric acid method in microplate format. Anal Biochem 339:69-72

21. Qi W, Fong C, Lamport DT (1991) Gum arabic glycoprotein is a twisted hairy rope: a new model based on O-galactosylhydroxyproline as the polysaccharide attachment site. Plant Physiol 96:848-855

22. Krause I, Bockhardt A, Neckermann H, Henle T, Klostermeyer H (1995) Simultaneous determination of amino acids and biogenic amines by reversed-phase high-performance liquid chromatography of the dabsyl derivatives. J Chromatogr A 715:67-79

23. Ai Y, Yu Z, Chen Y, Zhu X, Ai Z, Liu S, Ni D (2016) Rapid determination of the monosaccharide composition and contents in tea polysaccharides from Yingshuang green tea by pre-column derivatization HPLC. J Chem 2016:6065813. https://doi.org/10.1155/2016/6065813

24. Postulkova H, Chamradova I, Pavlinak D, Humpa O, Jancar J, Vojtova L (2017) Study of effects and conditions on the solubility of natural polysaccharide gum karaya. Food Hydrocoll 67:148-156

25. Huang Y, Lu J, Xiao C (2007) Thermal and mechanical properties of cationic guar gum/poly (acrylic acid) hydrogel membranes. Polym Degrad Stab 92:1072-1081

26. Tryfona T, Liang H-C, Kotake T, Tsumuraya Y, Stephens E, Dupree P (2012) Structural characterisation of Arabidopsis leaf arabinogalactan polysaccharides. Plant Physiol 160:653-666

27. Fadavi G, Mohammadifar MA, Zargarran A, Mortazavian AM, Komeili $R$ (2014) Composition and physicochemical properties of Zedo gum exudates from Amygdalus scoparia. Carbohydr Polym 101:1074-1080

28. Critto A, Carlon C, Marcomini A (2003) Characterization of contaminated soil and groundwater surrounding an illegal landfill (S. Giuliano, Venice, Italy) by principal component analysis and kriging. Environ Pollut 122:235-244 
29. Khatiy Chhetri DB (1999) Comparison of forest biomass across a humaninduced disturbance gradient in Nepal's Schima-Castanopsis forests. J Sustain For 9:69-82

30. Måren IE, Karki S, Prajapati C, Yadav RK, Shrestha BB (2015) Facing north or south: does slope aspect impact forest stand characteristics and soil properties in a semiarid trans-Himalayan valley? J Arid Environ 121:112-123

31. Rodrigues Filho G, de Assunção RM, Vieira JG, Meireles CdS, Cerqueira DA, da Silva Barud H, Ribeiro SJ, Messaddeq Y (2007) Characterization of methylcellulose produced from sugar cane bagasse cellulose: crystallinity and thermal properties. Polym Degrad Stab 92:205-210

32. Petrea P, Amarioarei G, Apostolescu N, Puiel AC, Ciovica S (2013) Some aspects of the characterization of vegetable gums: prunus persica (plum) and prunus domestica (cherry). Cellul Chem Technol 47:369-375

33. Cerqueira MA, Souza BW, Simões J, Teixeira JA, Domingues MRM, Coimbra MA, Vicente AA (2011) Structural and thermal characterization of galactomannans from non-conventional sources. Carbohydr Polym 83:179-185

34. Daoub RM, Elmubarak AH, Misran M, Hassan EA, Osman ME (2016) Characterization and functional properties of some natural Acacia gums. J Saudi Soc Agric Sci 17:241-249

35. Coates J (2000) Interpretation of infrared spectra, a practical approach. Ency Anal Chem 12:10815-10837

36. García del Moral LF, Rharrabti Y, Martos V, Royo C (2007) Environmentally induced changes in amino acid composition in the grain of durum wheat grown under different water and temperature regimes in a Mediterranean environment. J Agric Food Chem 55:8144-8151

37. Cao X, Ma Q, Zhong C, Yang X, Zhu L, Zhang J, Jin Q, Wu L (2016) Elevational variation in soil amino acid and inorganic nitrogen concentrations in Taibai Mountain, China. PloS One 11:e0157979

38. Song W, Yang R, Wu T, Wu C, Sun S, Zhang S, Jiang B, Tian S, Liu X, Han $T$ (2016) Analyzing the effects of climate factors on soybean protein, oil contents, and composition by extensive and high-density sampling in China. J Agric Food Chem 64:4121-4130

39. Gambetta JM, Cozzolino D, Bastian SE, Jeffery DW (2017) Exploring the effects of geographical origin on the chemical composition and quality grading of Vitis vinifera L. CV. Chardonnay grapes. Molecules 22:218

40. Amelung W, Zhang $X$, Flach K (2006) Amino acids in grassland soils: climatic effects on concentrations and chirality. Geoderma 130:207-217

41. Assefa Y, Bajjalieh N, Archontoulis S, Casteel S, Davidson D, Kovács P, Naeve S, Ciampitti IA (2018) Spatial characterization of soybean yield and quality (amino acids, oil, and protein) for United States. Sci Rep 8:14653
42. Sidle $A B$ (1967) Amino acid content of atmospheric precipitation. Tellus 19:128-135

43. Davis FW, Goetz S (1990) Modeling vegetation pattern using digital terrain data. Landsc Ecol 4:69-80

44. Austin M (2002) Spatial prediction of species distribution: an interface between ecological theory and statistical modelling. Ecol Modell 157:101-118

45. Lebourgeois $F$ (2007) Climatic signal in annual growth variation of silver fir (Abies alba Mill.) and spruce (Picea abies Karst.) from the French Permanent Plot Network (RENECOFOR). Ann For Sci 64:333-343

46. Li MH, Cherubini P, Dobbertin M, Arend M, Xiao WF, Rigling A (2013) Responses of leaf nitrogen and mobile carbohydrates in different Quercus species/provenances to moderate climate changes. Plant Biol 15:177-184

47. Sperling O, Silva LC, Tixier A, Théroux-Rancourt G, Zwieniecki MA (2017) Temperature gradients assist carbohydrate allocation within trees. Sci Rep 7:3265

48. Hanover JW (1966) Genetics of terpenes I. Gene control of monoterpene levels in Pinus monticola dougl. Heredity 21:73

49. Maxwell JC, Pesic P (2001) Theory of heat. Courier Corporation, North Chelmsford

50. Boer G, McFarlane N, Lazare M (1992) Greenhouse gas-induced climate change simulated with the CCC second-generation general circulation model. J Clim 5:1045-1077

51. Houghton J, Ding Y, Griggs D, Noguer M, Van Der Linden P, Dai X, Maskell K, Johnson C (2001) Climate change 2001: the scientific basis. Contribution of working group I to the third assessment report of the international panel on climate change. Cambridge University Press, Cambridge, vol 36, pp 44

52. Rezaei A, Nasirpour A, Tavanai H (2016) Fractionation and some physicochemical properties of almond gum (Amygdalus communis L.) exudates. Food Hydrocoll 60:461-469

53. Broido A (1969) A simple, sensitive graphical method of treating thermogravimetric analysis data. J Polym Sci B Polym Phys 7:1761-1773

54. Harnkarnsujarit N, Charoenrein S (2011) Influence of collapsed structure on stability of $\beta$-carotene in freeze-dried mangoes. Food Res Int 44:3188-3194

\section{Submit your manuscript to a SpringerOpen ${ }^{\circ}$ journal and benefit from:}

- Convenient online submission

- Rigorous peer review

- Open access: articles freely available online

- High visibility within the field

- Retaining the copyright to your article

Submit your next manuscript at $\boldsymbol{\nabla}$ springeropen.com 